\title{
Le consensus de Bellagio : Recommandations pour améliorer l'accès aux méthodes de contraception efficaces, réversibles à longue durée d'action
}

Population Council

Follow this and additional works at: https://knowledgecommons.popcouncil.org/departments_sbsr-rh

Part of the Demography, Population, and Ecology Commons, Family, Life Course, and Society Commons, International Public Health Commons, Medicine and Health Commons, and the Women's Health Commons How does access to this work benefit you? Let us know!

\section{Recommended Citation}

"Le consensus de Bellagio : Recommandations pour améliorer l'accès aux méthodes de contraception efficaces, réversibles à longue durée d'action." New York: Population Council, 2012. 


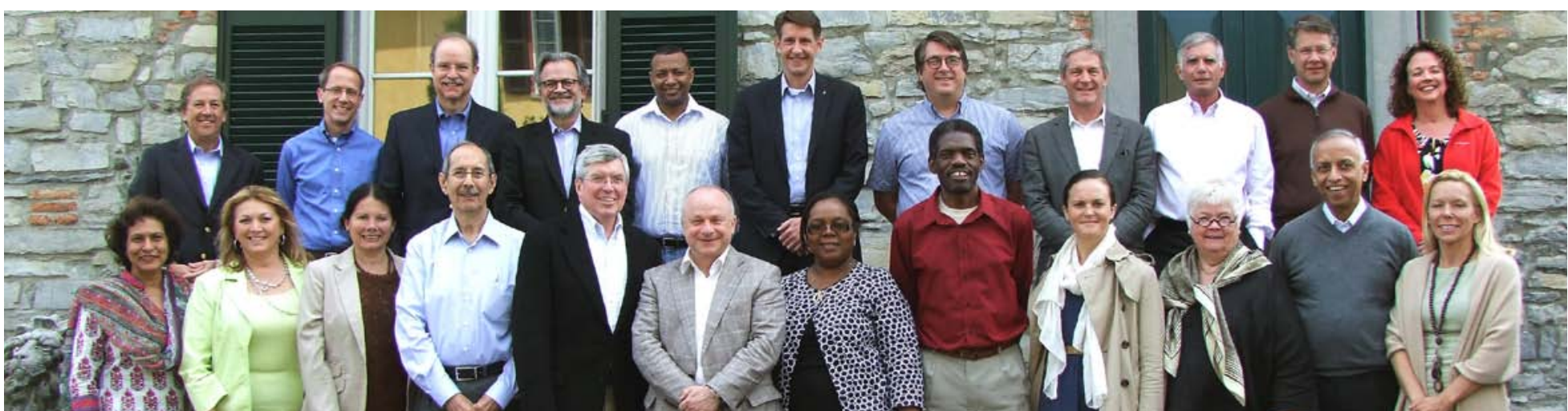

Des experts internationaux se réunissent pour discuter les possibilités d'accroître l'accès aux méthodes de contraception efficaces, réversibles à longue durée d'action (appelées $L A R C$ ). La réunion a été organisée par le Population Council, la Fédération internationale de gynécologie et d'obstétrique (FIGO), et la Coalition pour la fourniture de produits de santé reproductive (RHSC).

Plus de $\mathbf{2 0 0}$ millions de femmes dans le monde en développement souhaitent éviter une grossesse, mais n'utilisent aucune méthode de contraception moderne.

Pour beaucoup de ces femmes, en particulier les pauvres, d'importants obstacles structurels les empêchent d'accéder à l'éventail des méthodes de contraception disponibles. Le désir des femmes de retarder, d'espacer ou de limiter leurs grossesses est souvent combattu par leurs familles ou leurs communautés. Les programmes de planification familiale du secteur public, qui sont la principale source de contraception dans la plupart des pays en développement, offrent généralement des options limitées, et les produits contraceptifs ne sont pas toujours disponibles. Les prestataires de soins de santé ne sont peut-être pas formés à fournir les services et les conseils attendus par les femmes pour atteindre leurs objectifs en matière de reproduction. Compte tenu de ces facteurs, les femmes pauvres sont exposées à un risque important de grossesse non désirée, d'avortement et de mortalité et morbidité maternelle et infantile.

Les méthodes de contraception efficaces, réversibles à longue durée d'action (appelées LARC : Highly effective, long-acting, reversible contraceptives) telles que les dispositifs intra-utérins (DIU) et les implants contraceptifs sont souvent hors de portée pour les femmes qui vivent dans les pays en développement.

Parce qu'il est indispensable d'avoir un prestataire qualifié et parce que la faible priorité accordée à ces méthodes n'en fait pas une option viable pour les femmes, les programmes de planification familiale ne parviennent souvent pas à proposer aux femmes une gamme complète de méthodes, y compris celles qui sont les plus efficaces et les plus appropriées pour les femmes qui souhaitent retarder leur première grossesse ou leur prochaine grossesse ou limiter les naissances à venir.

Au niveau mondial, plus de 40 pour cent des grossesses ne sont pas planifiées. Les deux tiers de ces grossesses pourraient être évitées si les femmes ayant un besoin non satisfait en matière de contraception avaient accès à un choix de méthodes sûres et efficaces. Le fait d'avoir accès à différentes d'options et services contraceptifs basés sur un choix 
éclairé permet aux femmes de choisir la méthode la mieux adaptée à leurs besoins. Sachant que les méthodes de contraception efficaces, réversibles à longue durée d'action conservent leur efficacité pendant une longue période, les perturbations temporaires de la chaîne d'approvisionnement n'ont que peu d'impact sur la vie des utilisatrices. De même, les LARC coûtent généralement moins chères par mois d'utilisation que les méthodes contraceptives réversibles, qui sont moins efficaces. Par exemple, en raison de leur utilisation prolongée et plus efficace, les DIU au cuivre coûtent beaucoup moins chers par mois d'utilisation pour éviter une grossesse non désirée que les autres méthodes réversibles. En outre, le développement de nouveaux implants et l'amélioration des options de tarification des implants et des systèmes de contraception intra-utérins (SIU) rendront le coût de leur utilisation comparable à celui d'autres produits contraceptifs dans de nombreux marchés nationaux.

Depuis 1984, des accords internationaux ont réaffirmé le droit des femmes à décider du nombre et du moment d'avoir des enfants, ainsi qu'à leur proposer les moyens de réaliser leurs objectifs. L'amélioration de l'accès aux LARC, telles que les dispositifs intra-utérins (DIU ou SIU), aux implants, et, à l'avenir, à des contraceptifs injectables et des anneaux vaginaux à longue durée d'action permettrait de réduire de manière significative le nombre de grossesses non planifiées et d'améliorer la santé des femmes. Les LARC sont plus efficaces que d'autres méthodes contraceptives pour réduire la probabilité d'une grossesse non désirée. Par conséquent, une utilisation accrue des LARC permettrait de réduire le nombre de grossesses non désirées et d'avortements pratiqués dans des conditions dangereuses, et d'améliorer la santé maternelle et infantile. En plus de ces avantages pour la santé, le fait de répondre aux besoins contraceptifs des femmes permettrait d'accroître leurs opportunités économiques et leur contribution au bien-être de leur famille, ainsi qu'à la croissance économique nationale grâce à des revenus plus élevés par habitant.

Les besoins en LARC continueront de croître. En raison de la structure d'âge relativement jeune de la population dans les pays en développement, le nombre d'utilisatrices potentielles de méthodes contraceptives passera de 144 millions en 2005 à environ 214 millions en 2020. Parmi celles-ci, nous estimons que près de 57 millions de femmes chercheront à utiliser une LARC en 2020. Des travaux de recherche sur les politiques et les programmes ont montré que l'utilisation des LARC augmente lorsque les femmes disposent d'un plus grand choix de méthodes et que les questions relatives à l'équité sont la base des stratégies de prise en charge médicale. Le succès de ces programmes est bien la preuve qu'il faut poursuivre et élargir ces efforts.

\section{LA REUNION DE BELLAGIO}

En mai 2012, le Population Council, la Fédération internationale de gynécologie et d'obstétrique (FIGO), et la Coalition pour la fourniture de produits de santé reproductive (Reproductive Health Supplies Coalition - RHSC) ont organisé une réunion qui s'est tenue au Centre Bellagio de la Fondation Rockefeller pour se pencher sur les possibilités d'accroître l'accès aux LARC et accélérer les progrès vers la réalisation de l'Objectif du Millénaire pour le développement relatif à l'accès universel aux services de santé reproductive. Les participants, y compris les prestataires de services, les représentants des bailleurs de fonds, les décideurs politiques, les spécialistes de l'approvisionnement, les fabricants et les responsables de la santé publique, sont parvenus à un consensus sur l'analyse et les recommandations suivantes. 
L'accès à tous les contraceptifs, y compris aux LARC, doit être fondé sur le droit des femmes et des hommes à accéder à une gamme complète des services de santé reproductive. Les avantages incontestables de l'élargissement de l'accès des femmes aux LARC pour la santé publique et l'économie exigent des poli-
Nous sommes convaincus que les femmes sont capables de faire des choix éclairés sur la méthode contraceptive qui répond le mieux à leurs besoins, à condition que l'accès à ces méthodes soit encouragé, que des informations correctes soient disponibles et que la qualité des soins soit assurée.

tiques et des perspectives nouvelles. Le groupe a appelé à la mise en œuvre des actions suivantes pour assurer l'amélioration de l'accès à ces méthodes.

\section{RECOMMINDATIONS}

\begin{tabular}{|c|c|}
\hline $\begin{array}{l}\text { Modifier les } \\
\text { politiques : }\end{array}$ & $\begin{array}{l}\text { Les décideurs politiques doivent accorder une plus grande priorité aux efforts visant à ré- } \\
\text { duire les grossesses non désirées en répondant aux besoins non satisfaits en méthodes de } \\
\text { contraception efficaces, réversibles à longue durée d'action dans le cadre d'une gamme } \\
\text { complète d'options de méthodes. Cette priorité doit se refléter dans les politiques de finan- } \\
\text { cement, d'approvisionnement et de ressources humaines, ainsi que dans l'enregistrement, } \\
\text { l'introduction et la distribution des méthodes contraceptives. }\end{array}$ \\
\hline $\begin{array}{l}\text { Réduire les } \\
\text { coûts : }\end{array}$ & $\begin{array}{l}\text { Pour de nombreuses femmes vivant dans les pays en développement, les LARC ne sont ni } \\
\text { disponibles ni accessibles compte tenu des moyens financiers dont elles disposent. Pour } \\
\text { ces femmes, l'accès aux LARC dépend en grande partie des programmes financés par les } \\
\text { bailleurs de fonds et les gouvernements nationaux. Malgré le coût initial relativement élevé } \\
\text { des LARC, les gouvernements des pays en développement, en collaboration avec les bail- } \\
\text { leurs de fonds et les ONG, ont augmenté le niveau d'utilisation des LARC au cours des cinq } \\
\text { dernières années. Pour soutenir ces progrès, les fabricants, les donateurs internationaux et } \\
\text { les systèmes d'approvisionnement nationaux doivent collaborer pour élaborer des stratégies } \\
\text { permettant d'accroître l'accessibilité de l'achat et de l'offre de LARC. Ils doivent également } \\
\text { s'efforcer de maintenir un niveau d'approvisionnement fiable en LARC au sein des pays pour } \\
\text { permettre à ces femmes d'utiliser ces méthodes. Une plus grande disponibilité et utilisation } \\
\text { des LARC contribuera à accroître son accessibilité financière. }\end{array}$ \\
\hline $\begin{array}{l}\text { Intégrer les } \\
\text { LARC dans les } \\
\text { systèmes de } \\
\text { santé : }\end{array}$ & $\begin{array}{l}\text { Pour permettre aux femmes de choisir entre différentes catégories de produits, les systèmes } \\
\text { publics et privés de soins de santé doivent intégrer les LARC dans la gestion des services de } \\
\text { formation, de conseil, de prestation, de logistique et d'information. Parce que de nombreux } \\
\text { fournisseurs de soins après avortement et post-partum ont les compétences cliniques et de } \\
\text { conseil nécessaires pour introduire les LARC, une plus grande attention doit être accordée à } \\
\text { la fourniture des LARC dans le cadre des soins liés au post-partum et à l'avortement comme } \\
\text { l'un des moyens les plus simples d'améliorer l'accès des femmes qui en ont le plus besoin. }\end{array}$ \\
\hline $\begin{array}{l}\text { Assurer la } \\
\text { disponibilité et } \\
\text { l'accès: }\end{array}$ & $\begin{array}{l}\text { Les organisations qui œuvrent pour promouvoir un accès fiable aux méthodes contracep- } \\
\text { tives doivent assurer la disponibilité des méthodes contraceptives réversibles à longue du- } \\
\text { rée d'action sur les marchés publics et privés, et l'accessibilité des produits et des services } \\
\text { aux femmes les plus pauvres de chaque pays. Les gouvernements et les bailleurs de fonds } \\
\text { doivent promouvoir des choix éclairés en faisant la promotion d'une variété de choix contra- } \\
\text { ceptifs plutôt que de méthodes particulières. }\end{array}$ \\
\hline $\begin{array}{l}\text { Former et } \\
\text { superviser des } \\
\text { prestataires } \\
\text { qualifiés: }\end{array}$ & $\begin{array}{l}\text { Les responsables nationaux des programmes de planification familiale, de santé reproduc- } \\
\text { tive et de santé des femmes doivent mettre en place des systèmes de formation et de su- } \\
\text { pervision sur le lieu de travail basés sur les compétences pour assurer que du personnel } \\
\text { médical et paramédical qualifié soit habilité à fournir des conseils, à appliquer et à retirer les } \\
\text { LARC sur simple demande. }\end{array}$ \\
\hline $\begin{array}{l}\text { Solliciter l'appui } \\
\text { des associations } \\
\text { profession- } \\
\text { nelles : }\end{array}$ & $\begin{array}{l}\text { Les associations professionnelles, y compris la FIGO, la Confédération internationale des } \\
\text { sages-femmes, le Conseil international des infirmières, et d'autres associations nationales } \\
\text { et régionales du même type doivent travailler avec les gouvernements nationaux et les or- } \\
\text { ganisations du secteur privé pour former des prestataires médicaux et non médicaux, et } \\
\text { faciliter l'accès des femmes à l'information et aux services. }\end{array}$ \\
\hline
\end{tabular}




\begin{tabular}{|l|l|l|l}
\hline $\begin{array}{l}\text { Recueillir et } \\
\text { utiliser les } \\
\text { données afin } \\
\text { d'assurer } \\
\text { la qualité des } \\
\text { produits et } \\
\text { l'amélioration } \\
\text { des soins : }\end{array}$ & $\begin{array}{l}\text { Les données sur l'utilisation, la qualité des soins et l'efficacité des LARC et des autres mé- } \\
\text { thodes de planification familiale doivent être développées dans les pays à ressources limi- } \\
\text { tées ou, lorsqu'elles sont disponibles, diffusées à grande échelle. Ces données doivent être } \\
\text { révisées par l'OMS et par les associations professionnelles telles que la FIGO pour détermi- } \\
\text { ner la pertinence de recommandations supplémentaires sur les avantages liés à } \\
\text { l'élargissement de l'utilisation des LARC pour la santé publique. Des indicateurs doivent } \\
\text { être élaborés et déployés pour mesurer la facilité d'accès pour les femmes aux différentes } \\
\text { méthodes contraceptives de qualité, y compris les LARC. }\end{array}$ \\
\hline $\begin{array}{l}\text { Élaborer un plan } \\
\text { stratégique pour } \\
\text { atteindre ces } \\
\text { objectifs: }\end{array}$ & $\begin{array}{l}\text { Les représentants des gouvernements nationaux, les bailleurs de fonds, les fabricants de } \\
\text { contraceptifs, les responsables de programmes et les ONG, et les représentants des utilisa- } \\
\text { trices doivent se rencontrer avant la fin de l'année 2012 pour élaborer un plan stratégique } \\
\text { détaillé, fondé sur un diagnostic commun des obstacles les plus importants à l'accessibilité et } \\
\text { au coût abordable des méthodes de contraception efficaces, réversibles à longue durée d'ac- } \\
\text { tion. Le plan stratégique représente un outil pour augmenter les choix des femmes, assurer un } \\
\text { approvisionnement fiable, renforcer la qualité des services et améliorer la santé reproductive. }\end{array}$
\end{tabular}

La mise à disposition des LARC, des autres méthodes contraceptives et des soins liés à la santé reproductive doit être fondée sur des choix éclairés, le volontarisme et l'équité. Ces principes éthiques sont essentiels pour assurer que les besoins des femmes soient au cœur de toute discussion sur les technologies, et conformes aux plans d'action adoptés par la Conférence internationale sur la population et le développement (CIPD) en 1994, la Conférence sur les femmes en 1995, la Stratégie mondiale de l'OMS ayant trait à la santé génésique en 2004, et la Stratégie mondiale des Nations unies pour la santé de la femme et de l'enfant en 2010.

Nous souhaitons voir ces mesures pleinement mises en œuvre d'ici à 2020 avec des progrès significatifs compatibles avec la réalisation des Objectifs du Millénaire pour le développement d'ici 2015. Ces actions permettront d'améliorer la santé des femmes, d'élargir l'éventail de leurs opportunités et d'améliorer le bien-être de leurs familles et des communautés. En outre, les pays obtiendront les dividendes économiques dus à la baisse de la fécondité et une structure par âge avec de moins de dépendance et une plus grande participation au marché du travail au fil du temps. Nous demandons que ces recommandations soient intégrées à l'analyse et aux discussions qui se tiendront lors du Sommet sur la planification familiale le 11 juillet 2012, à Londres. Nous demandons également que les plans d'action issus de ce sommet tiennent compte des recommandations particulières visant à garantir un choix éclairé et l'équité, y compris un accès accru méthodes de contraception efficaces, réversibles à longue durée d'action dans le cadre d'une stratégie multi-méthodes plus large pour améliorer les options et la santé des femmes.

\section{LES PARTICIPANTS DE LA REUNION :}

(Rangée inférieure, de gauche à droite) : Shahida Zaidi, Ancien vice-président de la FIGO ; Turkiz Gokgol, Fondation Susan Thompson Buffett ; Laura Miranda, IPPF/WHR ; Anibal Faúndes, FIGO ; Peter Donaldson, Population Council ; Klaus Brill, Bayer Pharma AG ; Gloria Asare, Service de santé du Ghana, Ghana ; Roland Edgar Mhlanga, Ministère de la Santé, Afrique du Sud ; Julia Lowe, Fondation Bill \& Melinda Gates ; Jill Sheffield, Women Deliver ; Sabaratnam Arulkumaran, FIGO ; Saundra Pelletier, WomanCare Global. (Rangée supérieure, de gauche à droite) : Alan Staple, L'Initiative de la Fondation Clinton pour l'accès à la santé ; Jared Stamm, Population Council ; John Skibiak, Coalition pour la fourniture de produits de santé reproductive ; John Townsend, Population Council ; Aron Betru, Fondation des Nations unies ; Koen Kruytbosch, Merck/MSD ; Scott Radloff, USAID ; Ian Askew, Population Council ; James Trussell, Université de Princeton; Karl Hofmann, PSI ; Victoria Hale, Medicines360. (Absent de la photo : Eric Dupont, UNFPA)

Le Population Council s'attelle aux questions cruciales de la santé et du développement - qu'il s'agisse d'endiguer la progression du VIH, d'améliorer la santé reproductive ou d'offrir aux jeunes la promesse d'une vie satisfaisante et productive. Par ses travaux de recherche en biomédecine, en sciences sociales et en santé publique dans 50 pays, il s'efforce d'apporter avec ses partenaires des solutions qui conduisent à des politiques, des programmes et des technologies plus efficaces, pour améliorer la vie partout dans le monde. Fondé en 1952, le Population Council est une organisation non gouvernementale à but non lucratif. II siège à New York sous la conduite d'un conseil d'administration international. 